\title{
Log-concavity for series in reciprocal gamma functions and applications
}

\author{
S.I. Kalmykov and D.B. Karp*
}

\begin{abstract}
Euler's gamma function $\Gamma(x)$ is logarithmically convex on $(0, \infty)$. Additivity of logarithmic convexity implies that the function $x \rightarrow \sum f_{k} \Gamma(x+k)$ is also log-convex (assuming convergence) if the coefficients are non-negative. In this paper we investigate the series $\sum f_{k} \Gamma(x+k)^{-1}$, where each term is clearly log-concave. Log-concavity is not preserved by addition, so that non-negativity of the coefficients is now insufficient to draw any conclusions about the sum. We demonstrate that the sum is log-concave if $k f_{k}^{2} \geq(k+1) f_{k-1} f_{k+1}$ and is discrete Wright log-concave if $f_{k}^{2} \geq f_{k-1} f_{k+1}$. We conjecture that the latter condition is in fact sufficient for the log-concavity of the sum. We exemplify our general theorems by deriving known and new inequalities for the modified Bessel, Kummer and generalized hypergeometric functions and their parameter derivatives.
\end{abstract}

Keywords: Gamma function, log-concavity, Turán inequality, hypergeometric functions, modified Bessel function, Kummer function

MSC2010: 26A51, 33C20, 33C15, 33C05

1. Introduction. A positive continuous function $f$ defined on a real interval $I \subseteq \mathbf{R}$ is said to be Jensen log-concave if

$$
f(\mu+h)^{2} \geq f(\mu) f(\mu+2 h)
$$

for all $h>0$ and all $\mu$ such that $[\mu, \mu+2 h] \subseteq I$. If inequality (1) is strict the function $f$ is called strictly Jensen log-concave. If the sign of the inequality is reversed one talks about Jensen log-convexity (or strict Jensen log-convexity). For continuous functions Jensen log-concavity is equivalent to $\log$-concavity, i.e. concavity of $\log (f)$ (but is weaker in general). It is also equivalent to the seemingly stronger inequality

$$
\phi_{h, s}(\mu)=f(\mu+h) f(\mu+s)-f(\mu) f(\mu+h+s) \geq 0 \text { for all } h, s>0,
$$

which expresses the fact that $\mu \rightarrow f(\mu+h) / f(\mu)$ is non-increasing for any $h>0$. We tacitly assume here and below that all arguments lie in $I$. A function satisfying (2) is called Wright log-concave [13. Chapter I.4]. For comparisons of these notions and their higher order analogues see also the recent paper [15].

One is also frequently encountered with the situation when (11) or (2) only holds for integer values of $h$. We will express this fact by saying that $f$ is discrete log-concave or discrete Wright log-concave, respectively. In this case, however, we only have the implication (2) $\Rightarrow(1)$,

*Far Eastern Federal University and Institute of Applied Mathematics FEBRAS, Vladivostok, Russia. Emails: sergeykalmykov@inbox.ru (SK) and dimkrp@gmail.com (DK) 
while the reverse implication is not true even for continuous functions. We note that $f$ is discrete Wright log-concave if and only if

$$
\phi_{1, s}(x)=f(\mu+1) f(\mu+s)-f(\mu) f(\mu+s+1) \geq 0 \text { for all } s>0 .
$$

Indeed, (3) says that $f(\mu+1) / f(\mu)$ is non-increasing, so that $f(\mu+2) / f(\mu+1)$ is again nonincreasing implying that their product $f(\mu+2) / f(\mu)$ is non-increasing, i.e. satisfies (2) with $h=2$. In a similar fashion (2) holds for all integer values of $h$ which is discrete Wright log-concavity by definition. Discrete Jensen log-concavity and log-convexity are also frequently referred to as "Turán type inequalities" following the classical result of Paul Turán for Legendre polynomials [21.

If $f: \mathbb{N}_{0} \rightarrow \mathbf{R}_{+}$is a sequence, then discrete log-concavity reduces to the inequality $f_{k}^{2} \geq$ $f_{k-1} f_{k+1}, k \in \mathbb{N}$. We additionally require that the sequence $\left\{f_{k}\right\}_{k=0}^{\infty}$ is non-trivial and has no internal zeros: $f_{N}=0 \Rightarrow f_{N+i}=0$ for all $i \in \mathbb{N}_{0}$. Such sequences are also known as $P F_{2}$ (Pólya frequency sub two) or doubly positive [8].

Clearly, if $f$ is (Jensen or Wright) log-concave then $1 / f$ is (Jensen or Wright) log-convex. Notwithstanding the simplicity of this relation, several important properties of log-concavity and log-convexity differ. Particularly important is that log-convexity is additive while log-concavity is not. Further, log-convexity is a stronger property than convexity whereas log-concavity is weaker than concavity.

In [9] the second author and Sergei Sitnik initiated the investigation of the following problem: under what conditions on non-negative sequence $\left\{f_{k}\right\}$ and the numbers $a_{i}, b_{j}$ the function

$$
\mu \rightarrow f(\mu ; x):=\sum_{k=0}^{\infty} f_{k} \frac{\prod_{i=1}^{n} \Gamma\left(a_{i}+\mu+\varepsilon_{i} k\right)}{\prod_{j=1}^{m} \Gamma\left(b_{j}+\mu+\varepsilon_{n+j} k\right)} x^{k}
$$

is (discrete) log-concave or log-convex? Here $\Gamma$ is Euler's gamma function and $\varepsilon_{l}$ can be 1 or 0 . In [9] the authors treated the cases of (4) with $n=1, m=0, \varepsilon_{1}=1 ; n=m=1, \varepsilon_{1}=1, \varepsilon_{2}=0$; and $n=m=1, \varepsilon_{1}=0, \varepsilon_{2}=1$. Of course, the log-convexity cases are nearly trivial but the results of 9] go beyond log-convexity by showing that the function $\phi_{h, s}(\mu ; x)$ on the left-hand side of (2) has non-positive Taylor coefficients in powers of $x$, so that $x \rightarrow-\phi_{h, s}(\mu ; x)$ is absolutely monotonic. According to Hardy, Littlewood and Pólya theorem [14, Proposition 2.3.3] this also implies that this function is multiplicatively concave: $\phi_{h, s}\left(\mu ; x^{\lambda} y^{1-\lambda}\right) \geq \phi_{h, s}(\mu ; x)^{\lambda} \phi_{h, s}(\mu ; y)^{1-\lambda}$ for $\lambda \in[0,1]$.

In this paper we treat the case $n=0, m=1, \varepsilon_{1}=1$. We get slightly different results depending on conditions imposed on the sequence $\left\{f_{k}\right\}$. If $f_{k}$ is log-concave without internal zeros (i.e. doubly positive) we prove discrete Wright log-concavity of the series (44). We conjecture that the true logconcavity holds but we were unable to demonstrate this. If $\left\{f_{k} k\right.$ ! $\}$ is doubly positive we show that (4) is log-concave. We do so by establishing non-negativity of the Taylor coefficients of $\phi_{h, s}(\mu ; x)$ in powers of $x$ (either for all or only for integer $h>0$ ). Again, by Hardy, Littlewood and Pólya theorem this implies that $x \rightarrow \phi_{h, s}(\mu ; x)$ is multiplicatively convex.

The paper is organized as follows: in section 2 we collect several lemmas repeatedly used in the proofs; section 3 comprises two theorems constituting the main content of the paper; in section 4 we give applications to Bessel, Kummer and generalized hypergeometric functions and relate them to several previously known results.

2. Preliminaries. We will need several lemmas which we prove in this section. First, we formulate an elementary inequality we will repeatedly use below.

Lemma 1 Suppose $u, v, r, s>0, u=\max (u, v, r, s)$ and $u v>r s$. Then $u+v>r+s$.

Proof. Indeed, dividing by $u$ we can rewrite the required inequality as $r^{\prime}+s^{\prime}<1+v^{\prime}$, where $r^{\prime}=r / u \in(0,1), s^{\prime}=s / u \in(0,1), v^{\prime}=v / u \in(0,1)$. Since $r^{\prime} s^{\prime}<v^{\prime}$ by $r s<u v$, the required inequality follows from the elementary inequality $r^{\prime}+s^{\prime}<1+r^{\prime} s^{\prime}$. 
Lemma 1 is a particular case of a much more general result on logarithmic majorization - see [11, 2.A.b].

In the next lemma we say that a sequence has no more than one change of sign if the pattern is $(--\cdots--00 \cdots 00++\cdots++)$, where zeros and minus signs may be omitted.

Lemma 2 Suppose $\left\{f_{k}\right\}_{k=0}^{n}$ has no internal zeros and $f_{k}^{2} \geq f_{k-1} f_{k+1}, k=1,2, \ldots, n-1$. If the real sequence $A_{0}, A_{1}, \ldots, A_{[n / 2]}$ satisfying $A_{[n / 2]}>0$ and $\sum_{0 \leq k \leq n / 2} A_{k} \geq 0$ has no more than one change of sign, then

$$
\sum_{0 \leq k \leq n / 2} f_{k} f_{n-k} A_{k} \geq 0
$$

Equality is only attained if $f_{k}=f_{0} \alpha^{k}, \alpha>0$, and $\sum_{0 \leq k \leq n / 2} A_{k}=0$.

Proof. Suppose $f_{k}>0, k=s, \ldots, p, s \geq 0, p \leq n$. Log-concavity of $\left\{f_{k}\right\}_{k=0}^{n}$ clearly implies that $\left\{f_{k} / f_{k-1}\right\}_{k=s+1}^{p}$ is decreasing, so that for $s+1 \leq k \leq n-k+1 \leq p+1$

$$
\frac{f_{k}}{f_{k-1}} \geq \frac{f_{n-k+1}}{f_{n-k}} \Leftrightarrow f_{k} f_{n-k} \geq f_{k-1} f_{n-k+1}
$$

Since $k \leq n-k+1$ is true for all $k=1,2, \ldots,[n / 2]$, the weights $f_{k} f_{n-k}$ assigned to negative $A_{k} \mathrm{~s}$ in (5) are smaller than those assigned to positive $A_{k}$ s leading to (5). The equality statement is obvious.

Lemma 3 Suppose $m$ is non-negative integer. The inequality

$$
\sum_{k=0}^{m} \frac{1}{k !(m-k) !}\left(\frac{1}{\Gamma(k+\mu+a) \Gamma(m-k+\mu+b)}-\frac{1}{\Gamma(m-k+\mu) \Gamma(k+\mu+a+b)}\right) \geq 0
$$

holds for all $a, b \geq 0, \mu \geq-1$ and such that $\mu+a \geq 0, \mu+b \geq 0$. Equality is only attained if $a b=0$.

Proof. Using the easily verifiable relations

$$
(c)_{k}=\frac{\Gamma(c+k)}{\Gamma(c)}, \quad(m-k) !=\frac{(-1)^{k} m !}{(-m)_{k}} \text { and }(c)_{m-k}=\frac{(-1)^{k}(c)_{m}}{(1-c-m)_{k}}
$$

we obtain

$$
\begin{gathered}
\sum_{k=0}^{m} \frac{1}{k !(m-k) ! \Gamma(k+\alpha) \Gamma(m-k+\beta)}=\frac{1}{m ! \Gamma(\alpha) \Gamma(\beta)} \sum_{k=0}^{m} \frac{(-1)^{k}(-m)_{k}}{k !(\alpha)_{k}(\beta)_{m-k}} \\
=\frac{1}{m ! \Gamma(\alpha) \Gamma(\beta)(\beta)_{m}} \sum_{k=0}^{m} \frac{(-m)_{k}(1-\beta-m)_{k}}{k !(\alpha)_{k}}=\frac{(\alpha+\beta+m-1)_{m}}{m ! \Gamma(\alpha)(\alpha)_{m} \Gamma(\beta)(\beta)_{m}} \\
=\frac{\Gamma(\alpha+\beta+2 m-1)}{\Gamma(\alpha+m) \Gamma(\beta+m) \Gamma(\alpha+\beta+m-1) m !},
\end{gathered}
$$

where we have used the Chu-Vandermonde identity [2, Corollary 2.2.3]

$$
\sum_{k=0}^{m} \frac{(-m)_{k}(a)_{k}}{(c)_{k} k !}=\frac{(c-a)_{m}}{(c)_{m}}
$$


This leads to an explicit evaluation of the left hand side of (6) as

$$
\frac{\Gamma(2 \mu+a+b+2 m-1)}{\Gamma(2 \mu+a+b+m-1) m !}\left(\frac{1}{\Gamma(m+\mu+a) \Gamma(m+\mu+b)}-\frac{1}{\Gamma(m+\mu) \Gamma(m+\mu+a+b)}\right)
$$

For $a, b, \mu>0$, the required inequality reduces to log-convexity of $\Gamma(x)$ for $x>0$ [2, Corollary 1.2.6]. If $a b=0$ we clearly get the equality. If $a, b>0, \mu=m=0$, the second term in parentheses disappears and (6) holds strictly. If $m=0,-1 \leq \mu<0$ and $\mu+a \geq 0, \mu+b \geq 0$ then the term outside the parentheses reduces to 1 while the second term inside parentheses is strictly negative (since $\mu+a+b>0$ ), so that the sum is strictly positive. If $m \geq 1$ then $\mu+m \geq 0$ and we are back in the previous situation.

Lemma 4 Suppose $m \geq 0$ is an integer. Then for all complex $\beta$ and $\mu$

$$
\begin{array}{r}
S_{m}(\mu, \beta):=\sum_{k=0}^{m}\left\{\frac{1}{\Gamma(k+\mu+1) \Gamma(m-k+\mu+\beta)}-\frac{1}{\Gamma(k+\mu) \Gamma(m-k+\mu+\beta+1)}\right\} \\
=\frac{(\mu+\beta)_{m+1}-(\mu)_{m+1}}{\Gamma(\mu+m+1) \Gamma(\mu+\beta+m+1)} .
\end{array}
$$

Proof. We have

$$
\begin{aligned}
S_{m}(\mu, \beta)= & \frac{1}{\Gamma(\mu+1) \Gamma(\mu+\beta) \Gamma(\mu) \Gamma(\mu+\beta+1)} \sum_{k=0}^{m}\left\{\frac{\Gamma(\mu) \Gamma(\mu+\beta+1)}{(\mu+1)_{k}(\mu+\beta)_{m-k}}-\frac{\Gamma(\mu+1) \Gamma(\mu+\beta)}{(\mu)_{k}(\mu+\beta+1)_{m-k}}\right\} \\
= & \frac{1}{\Gamma(\mu+1) \Gamma(\mu+\beta+1)} \sum_{k=0}^{m}\left\{\frac{\mu+\beta}{(\mu+1)_{k}(\mu+\beta)_{m-k}}-\frac{\mu}{(\mu)_{k}(\mu+\beta+1)_{m-k}}\right\} \\
= & \frac{1}{\Gamma(\mu+1) \Gamma(\mu+\beta+1)} \sum_{k=0}^{m} \frac{1}{(\mu)_{k}(\mu+\beta)_{m-k}}\left\{\frac{\mu(\mu+\beta)}{\mu+k}-\frac{\mu(\mu+\beta)}{\mu+\beta+m-k}\right\} \\
= & \frac{1}{\Gamma(\mu+1) \Gamma(\mu+\beta+1)} \sum_{k=0}^{m} \frac{\beta+m-2 k}{(\mu+1)_{k}(\mu+\beta+1)_{m-k}} .
\end{aligned}
$$

Writing

$$
u_{k}=\frac{1}{(\mu+1)_{k-1}(\mu+\beta+1)_{m-k}}, 1 \leq k \leq m, \quad u_{0}=\frac{\mu}{(\mu+\beta+1)_{m}}, u_{m+1}=\frac{\mu+\beta}{(\mu+1)_{m}},
$$

we get a telescoping sum, since

$$
u_{k+1}-u_{k}=\frac{1}{(\mu+1)_{k}(\mu+\beta+1)_{m-k-1}}-\frac{1}{(\mu+1)_{k-1}(\mu+\beta+1)_{m-k}}=\frac{\beta+m-2 k}{(\mu+1)_{k}(\mu+\beta+1)_{m-k}}
$$

for $k=0,1, \ldots, m$, so that

$$
\sum_{k=0}^{m}\left(u_{k+1}-u_{k}\right)=u_{m+1}-u_{0}=\frac{\mu+\beta}{(\mu+1)_{m}}-\frac{\mu}{(\mu+\beta+1)_{m}}
$$

and

$$
\frac{1}{\Gamma(\mu+1) \Gamma(\mu+\beta+1)} \sum_{k=0}^{m} \frac{\beta+m-2 k}{(\mu+1)_{k}(\mu+\beta+1)_{m-k}}=\frac{(\mu+\beta)_{m+1}-(\mu)_{m+1}}{\Gamma(\mu+m+1) \Gamma(\mu+\beta+m+1)} .
$$

The following is a straightforward consequence of formula (7).

Corollary 1 If $\mu \geq-1, \beta \geq 0$ and $\mu+\beta \geq 0$ then $S_{m}(\mu, \beta) \geq 0$. The inequality is strict unless $\beta=0$. 
3. Main results. In this section we prove two general theorems for series in reciprocal gamma functions. The power series expansions in this section are understood as formal, so that no questions of convergence are discussed. In applications the radii of convergence will usually be apparent. The results of this section are exemplified by concrete special functions in the subsequent section.

Theorem 1 Suppose $\left\{f_{n}\right\}_{n=0}^{\infty}$ is a non-trivial non-negative log-concave sequence without internal zeros. Then the function

$$
\mu \mapsto f(\mu, x)=\sum_{n=0}^{\infty} \frac{f_{n} x^{n}}{n ! \Gamma(\mu+n)},
$$

is strictly log-concave on $(0, \infty)$ for each fixed $x \geq 0$. Moreover, the function

$$
\varphi_{a, b, \mu}(x):=f(a+\mu, x) f(b+\mu, x)-f(a+b+\mu, x) f(\mu, x)=\sum_{m=0}^{\infty} \varphi_{m} x^{m}
$$

has positive power series coefficients $\varphi_{m}>0$ for $\mu \geq-1, a, b>0$ and $\mu+a \geq 0, \mu+b \geq 0$ so that $\varphi_{a, b, \mu}(x)$ is absolutely monotonic and multiplicatively convex on $(0, \infty)$.

Proof. Cauchy product yields

$$
\varphi_{m}=\sum_{k=0}^{m} \frac{f_{k} f_{m-k}}{k !(m-k) !}\left(\frac{1}{\Gamma(k+\mu+a) \Gamma(m-k+\mu+b)}-\frac{1}{\Gamma(m-k+\mu) \Gamma(k+\mu+a+b)}\right) .
$$

Further, by Gauss summation (the first term is combined with the last, the second with the second last etc.) we can write $\varphi_{m}$ in the form

$$
\varphi_{m}=\sum_{k=0}^{[m / 2]} \frac{f_{k} f_{m-k}}{k !(m-k) !} M_{k}(a, b, \mu)
$$

where for $k<m / 2$

$$
\begin{aligned}
M_{k}(a, b, \mu)=\underbrace{[\Gamma(k+\mu+a) \Gamma(m-k+\mu+b)]^{-1}}_{=u}+\underbrace{[\Gamma(k+\mu+b) \Gamma(m-k+\mu+a)]^{-1}}_{=v} \\
-\underbrace{[\Gamma(m-k+\mu) \Gamma(k+\mu+a+b)]^{-1}}_{=r}-\underbrace{[\Gamma(k+\mu) \Gamma(m-k+\mu+a+b)]^{-1}}_{=s}
\end{aligned}
$$

and for $k=m / 2$

$$
M_{k}=[\Gamma(m / 2+\mu+a) \Gamma(m / 2+\mu+b)]^{-1}-[\Gamma(m / 2+\mu) \Gamma(m / 2+\mu+a+b)]^{-1} .
$$

Under assumptions on $\mu, a, b$ made in the theorem

$$
\sum_{k=0}^{[m / 2]} \frac{M_{k}(a, b, \mu)}{k !(m-k) !} \geq 0
$$

according to Lemma 3. Write $M_{k}:=M_{k}(a, b, \mu)$ for brevity. We aim to show that the sequence $\left\{M_{k}\right\}_{k=0}^{[m / 2]}$ has no more than one change of sign with $M_{[m / 2]}>0$ in order to apply Lemma 2. Due to log-convexity of $\Gamma(x)$ the ratio $x \mapsto \Gamma(x+\alpha) / \Gamma(x)$ is strictly increasing on $(0, \infty)$ when $\alpha>0$. This immediately implies $M_{[m / 2]}>0$ and the following inequalities

$$
v \geq u \Leftrightarrow \frac{\Gamma(m-k+\mu+b)}{\Gamma(m-k+\mu+a)} \geq \frac{\Gamma(k+\mu+b)}{\Gamma(k+\mu+a)}-\text { true for } k \leq m-k,
$$




$$
\begin{gathered}
u>s \Leftrightarrow \frac{\Gamma(m-k+\mu+a+b)}{\Gamma(m-k+\mu+b)}>\frac{\Gamma(k+\mu+a)}{\Gamma(k+\mu)}-\text { true for } k \leq m-k, \\
v \geq r \Leftrightarrow \frac{\Gamma(k+\mu+a+b)}{\Gamma(k+\mu+b)} \geq \frac{\Gamma(m-k+\mu+a)}{\Gamma(m-k+\mu)}-\text { true for }(m-b) / 2 \leq k, \\
r \geq v \Leftrightarrow \frac{\Gamma(m-k+\mu+a)}{\Gamma(m-k+\mu)} \geq \frac{\Gamma(k+\mu+a+b)}{\Gamma(k+\mu+b)}-\text { true for } k \leq(m-b) / 2 .
\end{gathered}
$$

If $(m-b) / 2 \leq k<m / 2$ then the sum of (12) and (13) yields $M_{k}=u+v-r-s>0$. If $k<(m-b) / 2$ then it follows from (11), (12) and (14) that $r>v>u>s$ (equality cannot be attained in (11) and (14) under this restriction on $k$ ). We will change notation to simplify writing: $\alpha=\mu, \beta=b+\mu, \delta=a$. According the hypothesis of the theorem we have $\beta>\alpha>0, \delta>0$. We will show now that if $M_{k}<0 \Leftrightarrow u+v<r+s$ for some $0<k<(m-b) / 2$ then $M_{k-1}<0$ as well. Indeed, using $z \Gamma(z)=\Gamma(z+1)$ we can write $M_{k-1}$ in the following form

$$
M_{k-1}(\delta)=\frac{k-1+\alpha+\delta}{m-k+\beta} u+\frac{k-1+\beta}{m-k+\alpha+\delta} v-\frac{k-1+\beta+\delta}{m-k+\alpha} r-\frac{k-1+\alpha}{m-k+\beta+\delta} s .
$$

Treating $u, v, r, s$ as constants we see that $M_{k-1}(0)<0$ by forming a combination of $r>v$ and $r+s>v+u$ with positive coefficients: $\left(C_{1}+C_{2}\right) r+C_{2} s>\left(C_{1}+C_{2}\right) v+C_{2} u$, where

$$
C_{1}+C_{2}=\frac{k-1+\beta}{m-k+\alpha}>C_{2}=\frac{k-1+\alpha}{m-k+\beta} .
$$

Further, differentiating with respect to $\delta$, we get

$$
M_{k-1}^{\prime}(\delta)=\frac{1}{m-k+\beta} u-\frac{k-1+\beta}{(m-k+\alpha+\delta)^{2}} v-\frac{1}{m-k+\alpha} r+\frac{k-1+\alpha}{(m-k+\beta+\delta)^{2}} s,
$$

which is obviously negative since $r>u$ (by (14) and (11) ) and $v>s$ (by (11) and (12)). This shows that $M_{k-1}<0$ and hence $\left\{M_{k}\right\}_{k=0}^{[m / 2]}$ has no more than one change of sign. Applying Lemma 2 with $A_{k}=M_{k} /(k !(m-k) !)$ we conclude that $\varphi_{m}>0$. Multiplicative convexity follows by Hardy, Littlewood and Pólya theorem [14, Proposition 2.3.3].

Remark 1. If $\left\{f_{n}\right\}_{n=0}^{\infty}$ is log-convex then $\varphi_{m}$ can take both signs.

Corollary 2 Assume the series in (8) converges for all $x \geq 0$. Then $\varphi_{a, b, \mu}(1 / y)$ is completely monotonic and log-convex on $[0, \infty)$, so that there exists a non-negative measure $\tau$ supported on $[0, \infty)$ such that

$$
\varphi_{a, b, \mu}(x)=\int_{[0, \infty)} e^{-t / x} d \tau(t) .
$$

Proof. According to [12, Theorem 3] a convergent series of completely monotonic with non-negative coefficients is again completely monotonic. This implies that $y \rightarrow \varphi_{a, b, \mu}(1 / y)$ is completely monotonic, so that the above integral representation follows by Bernstein's theorem [16, Theorem 1.4]. Log-convexity follows from complete monotonicity according to [14, Exersice 2.1(6)].

In the next corollary we adopt the convention $\Gamma(-1)=-\infty, \Gamma(0)=+\infty$.

Corollary 3 Under hypotheses and notation of Theorem 1

$$
\frac{\Gamma(a+\mu) \Gamma(b+\mu)}{\Gamma(\mu) \Gamma(a+b+\mu)}<\frac{f(\mu, x) f(a+b+\mu, x)}{f(a+\mu, x) f(b+\mu, x)}<1 \text { for all } x \geq 0 .
$$

If $\mu=0$ or $\mu=-1$ we additionally require that $x \neq 0$ otherwise the left inequality becomes equality. 
Proof. The estimate from above is a restatement of Theorem 1 since it is equivalent to $\phi_{\beta, \mu}(x)>0$.

The estimate from below is obvious for $\mu=-1$ for we have zero or negative number (if $a=1$ or $b=1$ ) on the left and a positive number on the right for $x>0$. The remaining proof will be divided into two cases (I) $\mu \geq 0$; and (II) $-1<\mu<0, \mu+a \geq 0, \mu+b \geq 0$ (recall that $a, b>0$ by hypotheses of Theorem [1).

In case (I) the left-hand inequality in (16) follows from strict log-convexity of $\mu \rightarrow \Gamma(\mu) f(\mu, x)$ which has been proved in [9, Theorem 3] (where one has to take account of the formula $(\mu)_{k}=$ $\Gamma(\mu+k) / \Gamma(\mu))$.

In case (II) $\Gamma(\mu)<0$ and the left-hand inequality in (16) reduces to

$$
\Gamma(a+\mu) f(a+\mu, x) \Gamma(b+\mu) f(b+\mu, x)>\Gamma(\mu) f(\mu, x) \Gamma(a+b+\mu) f(a+b+\mu, x) .
$$

This inequality follows by observing that $\Gamma(\mu) f(\mu, x)=\sum_{n=0}^{\infty} f_{n} x^{n}\left(n !(\mu)_{n}\right)^{-1}$ and

$$
\sum_{k=0}^{m} \frac{f_{k} f_{m-k}}{k !(m-k) !}\left\{\frac{1}{(a+\mu)_{k}(b+\mu)_{m-k}}-\frac{1}{(\mu)_{k}(a+b+\mu)_{m-k}}\right\}>0
$$

since for $k=1,2, \ldots, m(\mu)_{k}<0$ and for $k=0(b+\mu)_{m}<(a+b+\mu)_{m}$.

Corollary 4 Under hypotheses and notation of Theorem 1 and for all $x \geq 0$

$$
f(a+\mu, x) f(b+\mu, x)-f(a+b+\mu, x) f(\mu, x) \geq f_{0}^{2}\left[\frac{1}{\Gamma(\mu+a) \Gamma(\mu+b)}-\frac{1}{\Gamma(\mu) \Gamma(\mu+a+b)}\right]
$$

with equality only at $x=0$.

Proof. Indeed, the claimed inequality is just $\varphi_{a, b, \mu}(x) \geq \varphi_{a, b, \mu}(0)$ which is true by Theorem 1 .

Remark 2. Corollaries 3 and 4 imply by elementary calculation the following bounds for the so called "generalized Turanian" $\Delta_{\varepsilon}(\mu, x):=f(\mu, x)^{2}-f(\mu+\varepsilon, x) f(\mu-\varepsilon, x)$ :

$$
A_{\varepsilon}(\mu) f_{0}^{2} \leq \Delta_{\varepsilon}(\mu, x) \leq B_{\varepsilon}(\mu) f(\mu, x)^{2},
$$

where $\mu-\varepsilon \geq-1, \mu \geq 0, x \geq 0$ and

$$
A_{\varepsilon}(\mu)=\frac{\Gamma(\mu-\varepsilon) \Gamma(\mu+\varepsilon)-\Gamma(\mu)^{2}}{\Gamma(\mu-\varepsilon) \Gamma(\mu+\varepsilon) \Gamma(\mu)^{2}}, \quad B_{\varepsilon}(\mu)=\frac{\Gamma(\mu-\varepsilon) \Gamma(\mu+\varepsilon)-\Gamma(\mu)^{2}}{\Gamma(\mu-\varepsilon) \Gamma(\mu+\varepsilon)} .
$$

In particular, if $\varepsilon=1$ the bounds (17) simply to $(\mu \geq 0, x \geq 0)$

$$
\frac{f_{0}^{2}}{\mu \Gamma(\mu)^{2}} \leq f(\mu, x)^{2}-f(\mu+1, x) f(\mu-1, x) \leq \frac{1}{\mu} f(\mu, x)^{2}, \quad x \geq 0, \mu \geq 0 .
$$

Theorem 1 can be reformulated in terms of the numbers $g_{n}:=f_{n} / n$ !. The hypotheses of the theorem require then that these numbers satisfy

$$
g_{n}^{2} \geq \frac{n+1}{n} g_{n-1} g_{n+1}
$$

- a condition stronger then log-concavity. If we weaken it to log-concavity we are only able to prove discrete Wright log-concavity of $\mu \rightarrow f(\mu, x)$ in the next theorem. We conjecture below that the adjective "discrete" is actually redundant. 
Theorem 2 Suppose $\left\{g_{n}\right\}_{n=0}^{\infty}$ is a non-trivial non-negative log-concave sequence without internal zeros. Then the function

$$
\mu \rightarrow g(\mu, x)=\sum_{n=0}^{\infty} \frac{g_{n} x^{n}}{\Gamma(\mu+n)},
$$

is strictly discrete Wright log-concave on $(0, \infty)$ for each fixed $x \geq 0$. Moreover, the function

$$
\lambda_{\beta, \mu}(x):=g(\mu+1, x) g(\mu+\beta, x)-g(\mu, x) g(\mu+\beta+1, x)=\sum_{m=0}^{\infty} \lambda_{m} x^{m}
$$

has positive power series coefficients $\lambda_{m}>0$ for each $\mu \geq-1$ and $\beta>0$ such that $\mu+\beta \geq 0$. This implies that $x \rightarrow \lambda_{\beta, \mu}(x)$ is absolutely monotonic and multiplicatively convex on $(0, \infty)$.

Proof. Pursuing the same line of argument as in Theorem 1 we have by the Cauchy product and the Gauss summation:

$$
\lambda_{m}=\sum_{k=0}^{[m / 2]} g_{k} g_{m-k} M_{k}(1, \beta, \mu),
$$

where the numbers $M_{k}$ are defined in the proof of Theorem[1, below formula (9). Under assumptions on $\mu$ and $\beta$ made in the theorem we have

$$
\sum_{k=0}^{[m / 2]} M_{k}(1, \beta, \mu)=S_{m}(\mu, \beta)>0
$$

according to Corollary 1. Further, it has been shown in the course of the proof of Theorem 1 that the sequence $\left\{M_{k}\right\}_{k=0}^{[m / 2]}$ has no more than one change of sign with $M_{[m / 2]}>0$. Hence by Lemma 2 we conclude that $\lambda_{m}>0$ implying discrete Wright log-concavity of $\mu \rightarrow g(\mu, x)$ for each $x \geq 0$ and absolutely monotonicity of $x \rightarrow \lambda_{\beta, \mu}(x)$. Multiplicative convexity follows by Hardy, Littlewood and Pólya theorem [14, Proposition 2.3.3].

Corollary 5 Assume the series in (20) converges for all $x \geq 0$. Then $\lambda_{\beta, \mu}(1 / y)$ is completely monotonic and log-convex on $[0, \infty)$, so that there exists a non-negative measure $\tau$ supported on $[0, \infty)$ such that

$$
\lambda_{\beta, \mu}(x)=\int_{[0, \infty)} e^{-t / x} d \tau(t) .
$$

Corollary 6 Under hypotheses and notation of Theorem 2

$$
\frac{\mu}{\beta+\mu}<\frac{g(\mu, x) g(1+\beta+\mu, x)}{g(1+\mu, x) g(\beta+\mu, x)}<1 \text { for all } x \geq 0 \text {. }
$$

Proof. The estimate from above is a restatement of Theorem 2 since it is equivalent to $\lambda_{\beta, \mu}(x)>0$.

The estimate from below is obvious for $\mu=-1$ for we a negative number on the left and a positive number on the right. The remaining proof will be divided into two cases (I) $\mu \geq 0$; and (II) $-1<\mu<0, \mu+\beta \geq 0$ (recall that $\beta>0$ by hypotheses of Theorem 2).

In case (I) the left-hand inequality in (22) follows from strict log-convexity of $\mu \rightarrow \Gamma(\mu) g(\mu, x)$ which, in view of $(\mu)_{k}=\Gamma(\mu+k) / \Gamma(\mu)$, has been proved in [9, Theorem 3].

In case (II) the left-hand inequality in (22) can be rewritten as

$$
\Gamma(1+\mu) g(1+\mu, x) \Gamma(\beta+\mu) g(\beta+\mu, x)>\Gamma(\mu) g(\mu, x) \Gamma(1+\beta+\mu) g(1+b+\mu, x) .
$$


This inequality follows by observing that $\Gamma(\mu) g(\mu, x)=\sum_{n=0}^{\infty} g_{n}(\mu)_{n}^{-1}$ and

$$
\sum_{k=0}^{m} g_{k} g_{m-k}\left\{\frac{1}{(1+\mu)_{k}(\beta+\mu)_{m-k}}-\frac{1}{(\mu)_{k}(1+\beta+\mu)_{m-k}}\right\}>0,
$$

since $(\mu)_{k}<0$ for $k=1,2, \ldots, m$ and $(\beta+\mu)_{m}<(1+\beta+\mu)_{m}$ for $k=0$.

Corollary 7 Under hypotheses and notation of Theorem 2 and for all $x \geq 0$

$$
g(\mu+1, x) g(\mu+\beta, x)-g(\mu, x) g(\mu+\beta+1, x) \geq \frac{g_{0}^{2} \beta}{\Gamma(\mu+1) \Gamma(\mu+\beta+1)}
$$

with equality only at $x=0$.

Remark 3. Since we have only proved discrete Wright log-concavity in Theorem 2 we cannot make any statements about the "generalized Turanian" $g(\mu, x)^{2}-g(\mu+\varepsilon, x) g(\mu-\varepsilon, x)$. We can assert, however, that the standard Turanian satisfies the following bounds similar to those in (19)

$$
\frac{g_{0}^{2}}{\mu \Gamma(\mu)^{2}} \leq g(\mu, x)^{2}-g(\mu+1, x) g(\mu-1, x) \leq \frac{1}{\mu} g(\mu, x)^{2}, \quad x \geq 0, \mu \geq 0 .
$$

Conjecture 1. Under hypotheses of Theorem 2 the function $\mu \rightarrow g(\mu, x)$ is log-concave on $(0, \infty)$ for each fixed $x \geq 0$. Moreover, the function

$$
x \rightarrow g(\mu+\alpha, x) g(\mu+\beta, x)-g(\mu, x) g(\mu+\alpha+\beta, x)
$$

has positive power series coefficients for $\mu \geq-1$ and $\mu+\alpha \geq 0, \mu+\beta \geq 0$, where $\alpha, \beta>0$.

The above conjecture is equivalent to the assertion that

$$
\sum_{k=0}^{m}\left\{\frac{1}{\Gamma(k+\mu+\alpha) \Gamma(m-k+\mu+\beta)}-\frac{1}{\Gamma(k+\mu) \Gamma(m-k+\mu+\alpha+\beta)}\right\}>0
$$

which extends Corollary 1.

4. Applications and relation to other work. We start with the well-studied case of the modified Bessel function. Even for this classical case we can add to the current knowledge.

Example 1. The modified Bessel function is defined by the series [2, formula (4.12.2)]

$$
I_{\nu}(u)=\sum_{n \geq 0} \frac{(u / 2)^{2 n+\nu}}{n ! \Gamma(n+\nu+1)} .
$$

Hence, if we set $f_{n}=1 \forall n, x=(u / 2)^{2}$ and $\mu=\nu+1$ in Theorem 1 and use $\partial_{\nu}^{2} \log (u / 2)^{\nu}=0$ we immediately conclude that $\nu \rightarrow I_{\nu}(u)$ is log-concave on $(-1, \infty)$ for each fixed $u>0$. Moreover, for any $\nu \geq-1$ and $\nu-\varepsilon \geq-2$ the "generalized Turanian"

$$
u \rightarrow \Delta_{\varepsilon}(\nu, u):=\left(I_{\nu}(u)\right)^{2}-I_{\nu+\varepsilon}(u) I_{\nu-\varepsilon}(u)
$$

has positive power series coefficients, is multiplicatively convex and according to (17) satisfies

$$
(u / 2)^{2 \nu} A_{\varepsilon}(\nu+1) \leq \Delta_{\varepsilon}(\nu, u) \leq B_{\varepsilon}(\nu+1)\left(I_{\nu}(u)\right)^{2}, \quad u \geq 0,
$$


where $A_{\varepsilon}$ and $B_{\varepsilon}$ are defined in (18). In particular for $\varepsilon=1$ we get for $\nu \geq-1$ :

$$
\frac{(u / 2)^{2 \nu}}{(\nu+1) \Gamma(\nu+1)^{2}} \leq\left(I_{\nu}(u)\right)^{2}-I_{\nu+1}(u) I_{\nu-1}(u) \leq \frac{1}{\nu+1}\left(I_{\nu}(u)\right)^{2} .
$$

All the more, the function $(u / 2)^{-2 \nu} \Delta_{\varepsilon}(\nu, u)$ admits representation (15). Proofs of various forms of log-concavity of $I_{\nu}(u)$ have a long history. The discrete log-concavity, $I_{\nu-1}(x) I_{\nu+1}(x) \leq\left(I_{\nu}(x)\right)^{2}$, and the right-hand side of (26) for $\nu \geq 0$ were probably first demonstrated in 1951 by Thiruvenkatachar and Nanjundiah [20]. In fact, our method here is an extension of their approach, so that they could have proved the log-concavity of $\nu \rightarrow I_{\nu}(x)$. The discrete log-concavity was rediscovered by Amos [1] in 1974 and later by Joshi and Bissu [7] in 1991 with different proofs. Their paper also gives a proof of the right-hand side of (26) for $\nu \geq 0$. Finally, Lorch in [10] and later Baricz in [4] showed the log-concavity of $\nu \rightarrow I_{\nu}(x)$ on $(-1, \infty)$ and demonstrated the positivity of the function (24) for $\nu>-1 / 2$ and small $\varepsilon$. He also conjectured that the positivity remains true for $\nu>-1$ and $\varepsilon \in(0,1]$. Baricz [3] demonstrated the Lorch's conjecture for $\varepsilon=1$ and extended the right-hand side of (26) to $\nu>-1$. Our results here not only confirm Lorch's conjecture but also refine and strengthen it by proving (25) and the positivity of the power series coefficients of (24). Various extensions and a related results can also be found in [3, 5, 17]. We note that many proofs use special properties of the modified Bessel functions, like differential-recurrence relations, zeros etc. Theorem 1 and its corollaries show that it is in fact the structure of the power series that is responsible for the bounds (25) and (26).

Example 2. In his 1993 preprint [18] Sitnik, among other things, proved the inequality

$$
R_{n}^{2}(x)>R_{n-1}(x) R_{n+1}(x), \quad x>0, \quad n=1,2, \ldots,
$$

where

$$
R_{n}(x)=e^{x}-\sum_{k=0}^{n} \frac{x^{k}}{k !}=\frac{x^{n+1}}{(n+1) !}{ }_{1} F_{1}(1 ; n+2 ; x)
$$

is the exponential remainder. We can generalize this function as follows

$$
R_{\eta, \nu}(x)=\frac{x^{\nu+1}}{\Gamma(\nu+2)}{ }_{1} F_{1}(\eta ; \nu+2 ; x)=x^{\nu+1} \sum_{k=0}^{\infty} \frac{(\eta)_{k} x^{k}}{\Gamma(\nu+2+k) k !} .
$$

It is straightforward to check that the sequence $g_{k}=(\eta)_{k} / k$ ! is log-concave iff $\eta \geq 1$. Then according to Theorem 2 the function $\nu \rightarrow R_{\eta, \nu}(x)$ is discrete Wright log-concave on $(-2, \infty)$ for each fixed $\eta \geq 1, x>0$ and

$$
x \rightarrow R_{\eta, \nu+1}(x) R_{\eta, \nu+\beta}(x)-R_{\eta, \nu}(x) R_{\eta, \nu+\beta+1}(x)
$$

has positive power series coefficients for $\nu \geq-3, \nu+\beta \geq-2, \beta>0$. Moreover,

$$
\frac{x^{2 \nu+2}}{(\nu+2) \Gamma(\nu+2)^{2}} \leq R_{\eta, \nu}(x)^{2}-R_{\eta, \nu+1}(x) R_{\eta, \nu-1}(x) \leq \frac{1}{\nu+2} R_{\eta, \nu}(x)^{2}, \quad x \geq 0, \nu \geq-2 .
$$

Example 3. In addition to the results for the Kummer function presented in Example 2 above we can derive bounds for its logarithmic derivative. The logarithmic derivatives of the Kummer function plays an important role in some probabilistic applications - see [19]. Let us use abbreviated notation $F(a ; b ; x)={ }_{1} F_{1}(a ; b ; x)$. The following contiguous relations are easy to check (recall that $\left.F^{\prime}(a ; b ; x)=(a / b) F(a+1 ; b+1 ; x)\right)$ :

$$
a F(a ; b ; x)-a F(a+1 ; b ; x)+x F^{\prime}(a ; b ; x)=0,
$$




$$
\begin{gathered}
a b F(a+1 ; b ; x)=b(a+x) F(a ; b ; x)-(b-a) x F(a ; b+1 ; x), \\
b(b-1)(F(a ; b-1 ; x)-F(a ; b ; x))-a x F(a+1 ; b+1 ; x)=0 .
\end{gathered}
$$

Dividing (28) by $b$ and substituting $a F(a+1 ; b ; x)$ into (27) we get after simplification and dividing by $\Gamma(b+1)$ :

$$
\frac{1}{\Gamma(b+1)} F(a ; b+1 ; x)=\frac{F(a ; b ; x)-F^{\prime}(a ; b ; x)}{(b-a) \Gamma(b)}
$$

From (29) we obtain:

$$
\frac{1}{\Gamma(b-1)} F(a ; b-1 ; x)=\frac{1}{\Gamma(b)}\left(x F^{\prime}(a ; b ; x)+(b-1) F(a ; b ; x)\right)
$$

Thus we get the following expression for the Turanian:

$$
\begin{aligned}
\frac{F(a ; b ; x)^{2}}{\Gamma(b)^{2}} & -\frac{F(a ; b+1 ; x) F(a ; b-1 ; x)}{\Gamma(b+1) \Gamma(b-1)} \\
& =\frac{1}{\Gamma(b)^{2}(b-a)}\left\{-(a-1) F(a ; b ; x)^{2}+x F^{\prime}(a ; b ; x)^{2}+(b-x-1) F(a ; b ; x) F^{\prime}(a ; b ; x)\right\}
\end{aligned}
$$

Hence, inequality (19) becomes $(x>0, b>0, a \geq 1)$ :

$$
\frac{1}{b \Gamma(b)^{2}}<\frac{-(a-1) F(a ; b ; x)^{2}+x F^{\prime}(a ; b ; x)^{2}+(b-x-1) F(a ; b ; x) F^{\prime}(a ; b ; x)}{\Gamma(b)^{2}(b-a)}<\frac{1}{b \Gamma(b)^{2}} F(a ; b ; x)^{2},
$$

which leads to $\left(F \equiv F(a ; b ; x), F^{\prime} \equiv F^{\prime}(a ; b ; x)\right)$ :

$$
0<\frac{-(a-1)+x\left(F^{\prime} / F\right)^{2}+(b-x-1)\left(F^{\prime} / F\right)}{(b-a)}<\frac{1}{b} .
$$

Solving these quadratic inequalities we arrive at

$$
\frac{x+1-b+\sqrt{(x+1-b)^{2}+4 x(a-1)}}{2 x}<\frac{F^{\prime}(a ; b ; x)}{F(a ; b ; x)}<\frac{x+1-b+\sqrt{(x+1-b)^{2}+4 x a(b-1) / b}}{2 x}
$$

for $x>0$ and $b>a \geq 1$. The upper and lower bounds interchange if $x>0, a \geq 1$ and $0<b<a$ :

$$
\frac{x+1-b+\sqrt{(x+1-b)^{2}+4 x a(b-1) / b}}{2 x}<\frac{F^{\prime}(a ; b ; x)}{F(a ; b ; x)}<\frac{x+1-b+\sqrt{(x+1-b)^{2}+4 x(a-1)}}{2 x} .
$$

These bounds are quite precise numerically especially when $a$ and $b$ are close.

Example 4. The generalized hypergeometric function is defined by the series

$$
{ }_{p} F_{q}\left(\begin{array}{c}
a_{1}, a_{2}, \ldots, a_{p} \\
b_{1}, b_{2}, \ldots, b_{q}
\end{array} \mid z\right):=\sum_{n=0}^{\infty} \frac{\left(a_{1}\right)_{n}\left(a_{2}\right)_{n} \cdots\left(a_{p}\right)_{n}}{\left(b_{1}\right)_{n}\left(b_{2}\right)_{n} \cdots\left(b_{q}\right)_{n} n !} z^{n}
$$

where $(a)_{0}=1,(a)_{n}=a(a+1) \cdots(a+n-1), n \geq 1$, denotes the rising factorial. The series (30) converges in the entire complex plane if $p \leq q$ and in the unit disk if $p=q+1$. In the latter case its sum can be extended analytically to the whole complex plane cut along the ray $[1, \infty)[2$, Chapter 2]. Applications of Theorems 1 and 2 to generalized hypergeometric function is largely based on the following lemma. 
Lemma 5 Denote by $e_{k}\left(x_{1}, \ldots, x_{q}\right)$ the $k$-th elementary symmetric polynomial,

$$
e_{0}\left(x_{1}, \ldots, x_{q}\right)=1, \quad e_{k}\left(x_{1}, \ldots, x_{q}\right)=\sum_{1 \leq j_{1}<j_{2} \cdots<j_{k} \leq q} x_{j_{1}} x_{j_{2}} \cdots x_{j_{k}}, \quad k \geq 1 .
$$

Suppose $q \geq 1$ and $0 \leq r \leq q$ are integers, $a_{i}>0, i=1, \ldots, q-r, b_{i}>0, i=1, \ldots, q$, and

$$
\frac{e_{q}\left(b_{1}, \ldots, b_{q}\right)}{e_{q-r}\left(a_{1}, \ldots, a_{q-r}\right)} \leq \frac{e_{q-1}\left(b_{1}, \ldots, b_{q}\right)}{e_{q-r-1}\left(a_{1}, \ldots, a_{q-r}\right)} \leq \cdots \leq \frac{e_{r+1}\left(b_{1}, \ldots, b_{q}\right)}{e_{1}\left(a_{1}, \ldots, a_{q-r}\right)} \leq e_{r}\left(b_{1}, \ldots, b_{q}\right) .
$$

Then the sequence of hypergeometric terms (if $r=q$ the numerator is 1 ),

$$
f_{n}=\frac{\left(a_{1}\right)_{n} \cdots\left(a_{q-r}\right)_{n}}{\left(b_{1}\right)_{n} \cdots\left(b_{q}\right)_{n}}
$$

is log-concave, i.e. $f_{n-1} f_{n+1} \leq f_{n}^{2}, n=1,2, \ldots$ It is strictly log-concave unless $r=0$ and $a_{i}=b_{i}$, $i=1, \ldots, q$.

The proof of this lemma for $r=0$ can be found in [6, Theorem 4.4] and [9, Lemma 2]. The latter reference also explains how to extend the proof to general $r$ (see the last paragraph of [9]). This leads immediately to the following statements.

Theorem 3 Let $0 \leq p \leq q$ be integers. Denote

$$
f(\nu, x):=\frac{1}{\Gamma(\nu)}{ }_{p} F_{q+1}\left(a_{1}, \ldots, a_{p} ; \nu, b_{1}, \ldots, b_{q} ; x\right)
$$

and suppose that parameters $\left(a_{1}, \ldots, a_{p}\right),\left(b_{1}, \ldots, b_{q}\right)$ satisfy (31). Then the function $f(\nu, x)$ satisfies Theorem 1 and Corollaries 2 [4.

Theorem 4 Let $0 \leq p \leq q+1$ be integers. Denote

$$
g(\nu, x):=\frac{1}{\Gamma(\nu)}{ }_{p} F_{q+1}\left(a_{1}, \ldots, a_{p} ; \nu, b_{1}, \ldots, b_{q} ; x\right)
$$

and suppose that parameters $\left(a_{1}, \ldots, a_{p}\right),\left(1, b_{1}, \ldots, b_{q}\right)$ satisfy (31). Then the function $g(\nu, x)$ satisfies Theorem 2 and Corollaries 5.9 .

Example 5. Our last example is non-hypergeometric. Consider the parameter derivative of the regularized Kummer function:

$$
\frac{\partial}{\partial a} \frac{1}{\Gamma(b)}{ }_{1} F_{1}(a ; b ; x)=\sum_{k=0}^{\infty} \frac{(\psi(a+k)-\psi(a))(a)_{k}}{\Gamma(b+k) k !} x^{k}
$$

(this function cannot be expressed as hypergeometric function of one variable). If $a \geq 1$ then the sequence

$$
h_{k}=\frac{(\psi(a+k)-\psi(a))(a)_{k}}{k !}
$$

is log-concave, since

$$
\begin{aligned}
h_{k}^{2}- & h_{k-1} h_{k+1}=\frac{(a)_{k-1}(a)_{k}}{(k-1) ! k !} \times \\
& \quad\left\{\frac{a+k-1}{k}(\psi(a+k)-\psi(a))^{2}-\frac{a+k}{k+1}(\psi(a+k-1)-\psi(a))(\psi(a+k+1)-\psi(a))\right\}>0 .
\end{aligned}
$$


The last inequality holds because $y \rightarrow \psi(a+y)-\psi(a)$ is concave according to the Gauss formula 2 , Theorem 1.6.1]

$$
(\psi(a+y)-\psi(a))_{y}^{\prime \prime}=\psi^{\prime \prime}(a+y)=-\int_{0}^{\infty} \frac{t^{2} e^{-t(a+y)}}{1-e^{-t}} d t<0
$$

and hence is log-concave while $(a+k-1) / k>(a+k) /(k+1)$ if $a \geq 1$. By Theorem 2 this leads to

Theorem 5 Suppose $a \geq 1$. Then the function

$$
h(\nu, x):=\frac{\partial}{\partial a} \frac{{ }_{1} F_{1}(a ; \nu ; x)}{\Gamma(\nu)}
$$

satisfies Theorem 2 and Corollaries 5 . 7 .

5. Acknowledgements. We acknowledge the financial support of the Russian Basic Research Fund (grant 11-01-00038-a), Far Eastern Federal University and the Far Eastern Branch of the Russian Academy of Sciences.

\section{References}

[1] D.E. Amos, Computation of modified Bessel functions and their ratios, Math. Comp. vol.28, issue 25(1974), 239-251.

[2] G.E. Andrews, R. Askey and R. Roy, Special functions, Cambridge University Press, 1999.

[3] Á. Baricz, Turán type inequalities for modified Bessel functions, Bull. Aust. Math. Soc. 82 (2010), 254-264.

[4] Á. Baricz, Turán type inequalities for some probability density functions, Studia Scientiarum Mathematicarum Hungarica 47, 2(2010), 175-189.

[5] Á. Baricz, Bounds for Turánians of mofidied Bessel functions, preprint(2012), arXiv:1202.4853v1.

[6] V. Heikkala, M.K. Vamanamurthy, M. Vuorinen, Generalized Elliptic Integrals, Computational Methods and Function Theory, Volume 9, no.1, (2009), 75-109.

[7] C.M. Joshi and S.K. Bissu, Some inequalities of Bessel and modified Bessel functions, J. Austral. Math. Soc. Ser. A 50(1991), 333-342.

[8] S. Karlin, Total Positivity, Vol. I, Stanford University Press, California, 1968.

[9] D.B. Karp and S.M. Sitnik, Log-convexity and log-concavity of hypergeometric-like functions. Journal of Mathematical Analysis and Applications, vol. 364, no.2(2010), 384-394.

[10] L. Lorch, Monotonicity of the zeros of a cross product of Bessel functions, Meth. Appl. Anal. vol. 1(1994), 75-80.

[11] A.W. Marshall, I. Olkin and B.C. Arnold, Inequalities: Theory of Majorization and Its applications, second edition, Springer, 2011.

[12] K.S. Miller and S. Samko, Completely monotonic functions, Integr. Transf. and Spec. Funct. 12, 4(2001), 389-402. 
[13] D.S. Mitrinovć, J.E. Pecarić, A.M. Fink, Classical and new inequalities in Analysis, Kluwer Academic Publishers, 1993.

[14] C. P. Niculescu and L.-E. Persson, Convex Functions and Their Applications. A Contemporary Approach. Springer Science+Business Media, Inc, 2006.

[15] K. Nikodem, T. Rajba, S. Wa̧sowicz, On the classes of higher-order Jensen-convex functions and Wright-convex functions, preprint, 2012, arXiv:1201.4032 v1.

[16] R.L. Schilling, R. Song Z. Vondraček, Bernstein Functions. Theory and Applications, Walter de Gruyter, Studies in Mathematics, 37, 2010.

[17] J.Segura, Bounds for ratios of modified Bessel functions and associated Turán-type inequalities, J. Math. Anal. Appl. 374 (2011), 516-528.

[18] S.M. Sitnik, Inequalities for the exponential remainder, preprint, Institute of Automation and Control Process, FEBRAS, Vladivostok, 1993 (in Russian).

[19] S.Sra and D. Karp, The Multivariate Watson Distribution: Maximum-Likelihood Estimation and other Aspects, submitted to Journal of Multivariate Analysis, 2011, preprint: arXiv:1104.4422v2

[20] V.K. Thiruvenkatachar and T.S. Nanjundiah: Inequalities concerning Bessel functions and orthogonal polynomials, Proc. Indian Nat. Acad. Part A 33(1951), 373-384.

[21] Turán, P., On the zeros of the polynomials of Legendre, Časopis Pro Pěstování Matematiky, 75 (1950), 113-122. 